\title{
FACTORIZATION OF INVERTIBLE MATRICES OVER RINGS OF STABLE RANK ONE
}

\author{
LEONID N. VASERSTEIN AND ETHEL WHELAND
}

(Received 17 May 1989)

Communicated by R. Lidl

\begin{abstract}
Every invertible $n$-by- $n$ matrix over a ring $R$ satisfying the first Bass stable range condition is the product of $n$ simple automorphisms, and there are invertible matrices which cannot be written as the products of a smaller number of simple automorphisms. This generalizes results of Ellers on division rings and local rings.
\end{abstract}

1980 Mathematics subject classification (Amer. Math. Soc.) (1985 Revision): primary 15 A 23; secondary $20 \mathrm{H} 25$.

Keywords and phrases: stable rank, modules, simple matrices.

\section{Introduction}

In various situations it is instructive to represent a matrix as a product of matrices of a special nature. For example, every orthogonal $n$-by- $n$ matrix is the product of at most $n$ reflections [1], [2, Proposition 5, Chapter IX, $\S 6$, section 4] (see [4], for further work on reflections). In linear algebra, one writes an invertible matrix as a product of elementary matrices. One can ask how many elementary matrices (or commutators) are needed to represent any product of elementary matrices (respectively, commutators); see [3]. In multiplicative simplex methods, one writes an invertible matrix over a field as the product of matrices each of which differs from the identity matrix by one

The research was supported in part by NSF grant DMS 86-20428.

(C) 1990 Australian Mathematical Society 0263-6115/90 \$A2.00+0.00 
column. These matrices are simple in the sense of the following definition of Ellers [5].

An invertible matrix $\beta$ over a (possibly non-commutative) field $K$ is simple, if $\operatorname{rank}\left(\beta-1_{V}\right)=1$, that is, $\beta$ fixes every vector of some hyperplane in $V$. Examples of simple matrices include reflections, involutions, transvections, axial affinities and hyperreflections.

Motivated partly by geometric applications, Ellers showed that if $\beta$ is an element of $\operatorname{Aut}(V)$ and $\operatorname{rank}\left(\beta-1_{V}\right)=t$, there are simple mappings $\beta_{i}$ in $\operatorname{Aut}(V)$ such that $\beta=\beta_{1} \beta_{2} \cdots \beta_{t}$, and $t$ is the smallest number for which such a factorization of $\beta$ exists.

Later Ellers generalized these results to commutative local rings $R$ [6] and then to non-commutative local rings $R$ [7].

In this paper, we extend these results to any ring $R$ satisfying the first Bass stable range condition. Along with local rings $R$, this includes all semilocal rings $R$, all Artinian rings $R$, all 0-dimensional commutative rings $R$ (that is, every prime ideal of $R$ is maximal), and many other rings [8], [9], [12].

\section{Statement of results}

First, we introduce some definitions and notations.

Let $R$ be an associative ring with $1, V$ a right $R$-module,

$$
V^{*}=\operatorname{Hom}_{R}(V, R)
$$

the dual module, $\operatorname{End}(V)=\operatorname{Hom}_{R}(V, V)$ the ring of all $R$-linear endomorphisms of $V$, and $\operatorname{Aut}(V)$ the group of all automorphisms of $V(\operatorname{Aut}(V) \subset$ $\operatorname{End}(V))$. A vector $\nu \in V$ is called unimodular if $f \nu=1$ for some $f \in V^{*}$.

When $R$ is a division ring, the rank of $\alpha \in \operatorname{End}(V)$ is defined as the dimension of $\alpha V$. In general, there are different ways to extend the notion of rank. In this paper we use two different definitions of rank.

Definition 1. The rank, $\operatorname{rank}(\alpha)$, is the least integer $s \geq 0$ such that $\alpha=\nu_{1} f_{1}+\cdots+\nu_{s} f_{s}$ with $\nu_{i} \in V$ and $f_{i} \in V^{*}$.

In other words, $\alpha: V \rightarrow V$ can be decomposed as $V \rightarrow R^{S} \rightarrow V$, where $R^{S}$ is the $R$-module of $s$-columns over $R$.

DEFINITION 2. The unimodular rank, $u$-rank $(\alpha)$ is the least integer $s \geq 0$ such that $\alpha=\nu_{1} f_{1}+\cdots+\nu_{s} f_{s}$ with unimodular $\nu_{i} \in V$ and $f_{i} \in V^{*}$.

Both ranks could be infinite (when no such $s$ exists). Clearly, $\operatorname{rank}(\alpha) \leq$ $u$ - $\operatorname{rank}(\alpha)$ always. When $R$ is a division ring, both definitions coincide with the usual definition of the rank as the dimension of $\alpha V$.

An automorphism $\beta$ in $\operatorname{Aut}(V)$ is called simple (respectively, $u$-simple), if $\operatorname{rank}\left(\beta-1_{V}\right)=1$ (respectively, $u-\operatorname{rank}\left(\beta-1_{V}\right)=1$ ). That is, $\beta=1_{V}+\nu f$ 
with $\nu \in V$ ( $\nu$ is unimodular in the case of $u$-simple $\beta$ ) and $f \in V^{*}$. Invertibility of such $\beta$ is equivalent [10, Section 2] to $1+f \nu \in \mathrm{GL}_{1} R$.

Examples of simple automorphisms include transvections (when $f \nu=0$ ) and reflections (or involutions, when $f \nu=-2$ ). More generally, a hyperreflection can be defined [5] as a simple $\beta=1_{V}+\nu f$ with $f \nu$ having a finite order modulo the commutator subgroup [ $\left.\mathrm{GL}_{1} R, \mathrm{GL}_{1} R\right]$.

Recall that the first Bass stable range condition on $R$ is:

If $a, b \in R$ and $R a+R b=R$ then there is $c \in R$ such that $R(a+c b)=$ $R$.

We write $\operatorname{sr}(R)=1$ if $R$ satisfies this condition and $R \neq 0$. See [8], [9], [12] for various examples of such rings.

TheOREM 3. If $\operatorname{sr}(R)=1, \beta \in \operatorname{Aut}(V)$ and $\operatorname{rank}(\beta-1)=s<\infty$, then $\beta$ is the product of $s$ simple automorphisms, and it cannot be factored into any product of a smaller number of simple automorphisms.

THEOREM 4. If $\operatorname{sr}(R)=1, \beta \in \operatorname{Aut}(V)$ and $u-\operatorname{rank}(\beta-1)=s<\infty$, then $\beta$ is the product of $s$ u-simple automorphisms, and it cannot be factored into any product of a smaller number of $u$-simple automorphisms.

Theorem 3 will be proved in the next section. The proof of Theorem 4 is so similar that we leave it to the reader.

\section{Proof of Theorem 3}

Let $\mathrm{GL}_{n} R$ denote the group of all $n$-by- $n$ invertible matrices over $R$. It can be identified with $\operatorname{Aut}\left(R^{n}\right)$, where $R^{n}$ is the $R$-module of $n$-columns over $R$.

Lemma 5. Assume that $\operatorname{sr}(R)=1$. Let $n \geq 1$ be an integer, and $\beta=\left(b_{i, j}\right) \in \mathrm{GL}_{n} R$. Then there is a simple matrix $\gamma \in \mathrm{GL}_{n} R$ such that $\left(\gamma \beta \gamma^{-1}\right)_{n, n} \in \mathrm{GL}_{1} R$.

Proof. Consider the last row $\left(b_{n, 1}, \ldots, b_{n, n}\right)$ of the matrix $\beta=\left(b_{i, j}\right) \in$ $\mathrm{GL}_{n} R$. Since $\beta$ is invertible $\sum b_{n, i} R=R$. The first Bass stable range condition implies all higher Bass conditions for $R$ as well as for the opposite ring [11]. So there are $c_{i} \in R$ such that

$$
\left(b_{n, n}+b_{n, 1} c_{1}+\cdots+b_{n, n-1} c_{n-1}\right) R=R .
$$

Since $\operatorname{sr}(R)=1$, every one-sided unit in $R$ is a unit (a result of Kaplansky, see [12]). So $b_{n, n}+b_{n, 1} c_{1}+\cdots+b_{n, n-1} c_{n-1} \in \mathrm{GL}_{1} R$. Let $\gamma$ be the simple 
matrix which differs from the identity matrix $1_{n}$ only in the last column, the entries of the last column of $\gamma$ being $-c_{1},-c_{2}, \ldots,-c_{n-1}, 1$. Then $\left(\gamma \beta \gamma^{-1}\right)_{n, n}=b_{n, n}+b_{n, 1} c_{1}+\cdots+b_{n, n-1} c_{n-1} \in \mathrm{GL}_{1} R$.

Let us prove now the first conclusion of Theorem 3. So let $\beta=1_{V}+$ $\nu_{1} f_{1}+\cdots+\nu_{s} f_{s} \in \operatorname{Aut}(V)$ with $\nu_{i} \in V$ and $f_{i} \in V^{*}$. We want to prove that $\beta$ is a product of $s$ simple matrices. We proceed by induction on $s$. Set $b_{i, j}=f_{i} \nu_{j} \in R$ and consider the matrix $\beta^{\prime}=1_{s}+\left(b_{i, j}\right)$. By $[10$, Section 2], $\beta^{\prime} \in \mathrm{GL}_{s} R$. By Lemma 5 above, there is $\gamma \in \mathrm{GL}_{s} R$ such that $\left(\gamma \beta^{\prime} \gamma^{-1}\right)_{s, s} \in \mathrm{GL}_{1} R$. Replacing $\left(\nu_{1}, \ldots, \nu_{s}\right)$ by $\left(\nu_{1}, \ldots, \nu_{s}\right) \gamma^{-1}$ and $\left(f_{1}, \ldots, f_{s}\right)^{\top}$ by $\gamma\left(f_{1}, \ldots, f_{s}\right)^{\top}$, we do not change $\beta$, but replace $\beta^{\prime}=$ $1+s+\left(f_{1}, \ldots, f_{s}\right)^{\top}\left(\nu_{1}, \ldots, \nu_{s}\right)$ by $\gamma \beta^{\prime} \gamma^{-1}$. So we can assume that $1+f_{s} \nu_{s}=$ $\left(\beta^{\prime}\right)_{s, s} \in \mathrm{GL}_{1} R$. By [10, Section 2], $\delta=1_{V}+\nu_{s} f_{s} \in \operatorname{Aut}(V)$. So $\delta$ is a simple matrix. We have $\beta=\delta\left(1_{V}+\left(\delta^{-1} \nu_{1}\right) f_{1}+\cdots+\left(\delta^{-1} \nu_{s-1}\right) f_{s-1}\right)$. By the induction hypothesis, the second factor, $\left(1_{V}+\left(\delta^{-1} \nu_{1}\right) f_{1}+\cdots+\left(\delta^{-1} \nu_{s-1}\right) f_{s-1}\right)$ is the product of $s-1$ simple automorphisms. So $\beta$ is the product of $s$ simple automorphisms.

Let us prove now the second conclusion of Theorem 3. That is, we want to prove that if $\beta=\delta_{1} \cdots \delta_{t}$ is the product of $t$ simple automorphisms $\delta_{i}$, then $\operatorname{rank}\left(\beta-1_{V}\right) \leq t$. We write $\delta_{i}=1_{V}+\nu_{i} f_{i}$ with $\nu_{i} \in V$ and $f_{i} \in V^{*}$. By induction on $m$, we see easily that $\delta_{1} \cdots \delta_{m}=1_{V}+\nu_{1} g_{1}+\cdots+\nu_{m} g_{m}$, where $g_{i} \in V^{*}$ depend on $m$. So $\operatorname{rank}\left(\beta-1_{V}\right) \leq t$.

Theorem 3 is proved. We complement it with the following result.

Proposition 6. For any associative ring $R$ with $\operatorname{sr}(R)=1$, any integer $n \geq 2$, and any integer $s$ in the interval $0 \leq s \leq n$, there is a matrix $\beta \in \mathrm{GL}_{n} R$ with $u-\operatorname{rank}\left(\beta-1_{n}\right)=\operatorname{rank}\left(\beta-1_{n}\right)=s$. So this $\beta$ is the product of $s$ simple matrices and it is not a product of a smaller number of simple matrices.

To prove this proposition we will need the following two lemmas.

LEMMA 7. Let $R$ be an associative ring with $\operatorname{sr}(R)=1$ and $\alpha \in \operatorname{End}(V)$ be such that the $R$-module $\alpha V$ is a direct summand of $V$ and has a free basis of cardinality $s$. Then $u-\operatorname{rank}(\alpha)=\operatorname{rank}(\alpha)=s$.

Proof. Let $\left\{e_{j}\right\}$ be a free basis for $\alpha V$ of cardinality $s$.

We prove first that $\operatorname{rank}(\alpha) \leq u-\operatorname{rank}(\alpha) \leq s$. If $s=\infty$, there is nothing to prove, so let $s<\infty$. For every $\nu$ in $V$, we have $\alpha \nu=\sum e_{i} f_{i}(\nu)$ with $f_{i}(\nu) \in R$. Since $\left\{e_{i}\right\}$ is a basis, $f_{i} \in V^{*}$. So $\alpha=\sum e_{i} f_{i}$, hence 
$\operatorname{rank}(\alpha) \leq u-\operatorname{rank}(\alpha) \leq s$. (Note that $\operatorname{rank}(\alpha) \leq s$ holds even without the assumption that $\alpha V$ is a direct summand.)

Let us prove now that $\operatorname{rank}(a) \geq s$. Suppose on the contrary that $t=$ $\operatorname{rank}(\alpha)<s$. That is,

$$
\alpha=\nu_{1} f_{1}+\cdots+\nu_{t} f_{t} \in \operatorname{Aut}(V)
$$

with $\nu_{i} \in V$ and $f_{i} \in V^{*}$. Pick $\pi \in \operatorname{End}(V)$ such that $\pi^{2}=\pi$ and $\pi V=\alpha V$. Set $u_{i}=\pi \nu_{i}$. We can write $u_{i}=\sum_{j} e_{j} a_{j, i}$ with $a_{j, i} \in R$. Note that $t<\infty$, so only finitely many $e_{j}$ are involved in all these linear combinations. Say, $u_{i}=\sum_{j}^{m} e_{j} a_{j, i}$ for $i=1, \ldots, t$ with $t<m<\infty$. Now we write $e_{j}=\sum u_{i} b_{i, j}$ for $j=1, \ldots, m$ with $b_{i, j} \in R$. We have $\alpha \beta=1_{m}$, where $\alpha=\left(a_{j, i}\right)$ and $\beta=\left(b_{i, j}\right)$. Complementing $\alpha$ by zero columns and $\beta$ by zero rows, we obtain two matrices $\alpha^{\prime}, \beta^{\prime}$ in the ring $M_{m} R$ of square matrices over $R$ such that $\alpha^{\prime} \beta^{\prime}=\alpha \beta=1_{m}$. Since $\operatorname{sr}(R)=1$, we have $\operatorname{sr}\left(M_{n} R\right)=1$ by [11]. So, by Kaplansky's result [12], $\beta \in \mathrm{GL}_{m} R$. But since $\beta$ has a zero row, this is impossible.

ReMARK. Lemma 7 holds if the condition $\operatorname{sr}(R)=1$ is replaced by the condition $R \neq 0$ together with the condition $\operatorname{sr}(R)<\infty$ or the condition that $R$ is commutative.

LeMma 8. For any $n \geq 2$ there exists an invertible matrix $\beta_{n}$ in $\mathrm{GL}_{n} R$ such that the matrix $\beta_{n}-1$ is also invertible.

Proof. When $n=2$, we can take

$$
\beta_{2}=\left(\begin{array}{rr}
1 & -1 \\
1 & 0
\end{array}\right)
$$

When $n=3$, we can take

$$
\beta_{3}=\left(\begin{array}{lll}
1 & 0 & 1 \\
1 & 1 & 0 \\
0 & 1 & 0
\end{array}\right) .
$$

For $n \geq 4$, we can write $\beta_{n}$ as the direct sum of the above matrices $\beta_{2}$ and $\beta_{3}$. For example, $\beta_{4}=\beta_{2} \oplus \beta_{2}$ is the required matrix in $\mathrm{GL}_{4} R$, $\beta_{5}=\beta_{3} \oplus \beta_{2}$ is the required matrix in $\mathrm{GL}_{5} R$, and so on.

Proof of Proposition 6. When $s=0$, we take $\beta=1_{n}$. When $1 \leq s \leq$ $n-1$, we can take $\beta=\gamma+1_{n-s-1}$, where $\gamma \in \mathrm{GL}_{s+1} R$ is the Jordan matrix with ones along the diagonal. Then $\left(\beta-1_{n}\right) R^{n}$ is a direct summand of $R^{n}$ with $s$ free generators, so $u-\operatorname{rank}\left(\beta-1_{n}\right)=\operatorname{rank}\left(\beta-1_{n}\right)=s$ by Lemma 7 . 
Finally, when $s=n$, we find $\beta$ as in Lemma 8 , so $\left(\beta-1_{n}\right) R^{n}=R^{n}$, hence $\operatorname{rank}\left(\beta-1_{n}\right)=n$ by Lemma 7 .

\section{References}

[1] E. Artin, Geometric algebra, (Wiley-Interscience, New York, 1957).

[2] N. Bourbaki, Éléments de mathématique, Livre 2 (Algèbre, Hermann, Paris, 1947).

[3] R. K. Dennis and L. N. Vaserstein, 'On a question of M. Newman on the number of commutators', J. Algebra 118 (1988), 150-161.

[4] D. Ż. Djoković and J. Malzan, 'Products of reflections in the general linear group over a division ring', Linear Algebra Appl. 28 (1979), 53-62.

[5] E. W. Ellers, 'Product of axial affinities and products of central collineations', in The Geometric Vein, pp. 465-470, (Springer, New York, 1982).

[6] E. W. Ellers and H. Ishibashi, 'Factorization of transformations over a local ring', Linear Algebra 85 (1987), 17-27.

[7] E. W. Ellers and H. Lausch, 'Length theorems for the general linear group of a module over a local ring', J. Austral. Math. Soc. Ser. A 46 (1989), 122-131.

[8] K. R. Goodearl and P. Menal, 'Stable range one for rings with many units', J. Pure Appl. Algebra 54 (1988), 261-287.

[9] I. F. Putnam, 'The invertible elements are dense in the irrational rotation $C^{*}$-algebras', preprint.

[10] L. N. Vaserstein, ' $K_{1}$-theory and the congruence subgroup problem', Mat. Zametki 5 (1969), 233-244 (Translation, Math. Notes 5, 141-148).

[11] L. N. Vaserstein, 'The stable range of rings and the dimension of topological spaces', Funkcional. Anal. i Priložen. 5 (1971), 17-27 (Translation, Functional Anal. Appl. 5, 102-110).

[12] L. N. Vaserstein, 'Bass's first stable range condition', J. Pure Appl. Algebra 34 (1984), 319-330.

The Pennsylvania State University

University Park, Pennsylvania 16802

U.S.A. 